\title{
Hydrocephalus nach Dura- und Gehirnblutungen bei Säuglingen.
}

\author{
Von \\ Dr. Walter Fischer, \\ Privatdozent und 1. Assistent am pathol. Institut Götingen. \\ (Aus dem pathologischen Institut Freiburg i. B.) \\ (Eingegangen am 2\%. April 1911.)
}

Vor Kurzem hat Beneke1) ${ }^{2}$ ) über Zerreißungen des Tentoriums bei der Geburt berichtet und kurz auf einige Folgezustände solcher Geburtstraumen hingewiesen. Tch möchte in folgendem einen weiteren Beitrag zur Kenntnis von Gehirnveränderungen bringen, die bei kleinen Kindern nach Traumen entstanden sind. Es handelt sich in den beiden mitzuteilenden Fällen um ausgedehnte Hirnblutungen und Hydrocephalus bei Säuglingen. Die Krankengeschichten bieten in beiden Fällen manches Interessante und seien hier mit einigen Kürzungen mitgeteilt.

\section{Fall.}

Eine 26jährige, völlig gesunde Frau wird als 1. para in die Freiburger Frauenklinik aufgenommen. Wehenbeginn am 24. III. 1909 I Uhr morgens. Um $9 \mathrm{Uhr}$ früh wird eine 1. Schädellage festgestellt, der Kopf des Kindes beweglich über dem Becken. Das Becken ist leicht verengt. Skopolamin-Morphium-Dämmerschlaf. $9 \mathrm{Uhr}$ abends erfolgt die Geburt eines $2900 \mathrm{~g}$ schweren, $52 \mathrm{~cm}$ langen Knaben, mit den Zeichen der Reife. Eine größere Kopfgeschwulst ist nicht vorhanden.

Es wurden folgende Maße des kindlichen Schädels festgestellt:

1) Beneke, Über Tentoriumzerreißungen bei der Geburt. Verhandl. d. Deutsch. pathol. Gesellschaft 14, 128. 1910.

2) Beneke, Über Tentoriumzerreißungen bei der Geburt, sowie die Bedeutung der Duraspannung für chron. Gehirnerkrankungen. Münch. med. Wochenschrift 1910. Nr. 41. 


\begin{tabular}{|c|c|c|c|c|c|c|}
\hline \multirow[b]{2}{*}{ Diameter } & \multirow[b]{2}{*}{ - bitemporalis } & & & & normal & \multirow[t]{2}{*}{$\begin{array}{l}\text { bei Entlassung } \\
\text { 2. IV. } 09\end{array}$} \\
\hline & & ${ }^{\circ}$ & 8 & $\mathrm{em}$ & 8 & \\
\hline ," & biparietalis & . & $91 / 2$ & & $9^{1 / 4}$ & 11,0 \\
\hline , & suboccipito-bregmaticus & . & $9^{1} / 4$ & & $91 / 2$ & \\
\hline & suboccipito-frontalis. . & . & $121 / 2$ & & 12 & \\
\hline , & fronto-occipitalis & . & 14 & , & 12 & 12,5 \\
\hline \multicolumn{2}{|c|}{ Circumferentia suboccipito-frontalis } & . & 34 & $"$ & 34 & \\
\hline 3 & suboccipito-bregmaticus & . . & 32 & $"$ & & \\
\hline, & fronto-occipitalis . . & . . & 38 & ", & 34 & \\
\hline
\end{tabular}

Das Wochenbett verlief ohne Störung; Mutter und Kind wurden nach 9 Tagen, am 2. IV., entlassen.

Irgendwelche Abnormitäten des kindlichen Schädels oder cerebrale Erscheinungen wurden bei dem Kinde während des Aufenthaltes in der Klinik nicht wahrgenommen.

Nach Entlassung der Mutter aus der Klinik hat die Mutter das Kind angeblich noch 2 Wochen gestillt. Etwa seit Mitte April bemerkt die Mutter bei dem Kinde ein abnorm starkes Wachstum des Kopfes; bei der Geburt sei er ganz normal gewesen.

Das Kind wird am 23. IV. in das Hildaspital aufgenommen.

Der Krankengeschichte entnehme ich folgendes:

Kleines, unruhiges, blasses, sehr mageres Kind, dessen Körper in auffälligem Gegensatz zu dem riesengroßen Kopf steht (Kopfumfang 44,5 cm). Körpergewicht $3090 \mathrm{~g}$. Haut welk, faltenreich. Fettpolster fehlt völlig. Die Stirn stark vorgebuchtet. Die große Fontanelle erstreckt sich mit ihrer vorderen Spitze bis $1 \mathrm{~cm}$ oberhalb der Nasenwurzel, nach hinten geht sie direkt in die kleine Fontanelle über. Links seitlich reicht die große Fontanelle bis $2 \mathrm{~cm}$ oberhalb des linken Ohres, rechts seitlich bis $3 \frac{1}{2} \mathrm{~cm}$ oberhalb des rechten Ohres. Die Nase zeigt Sattelform. Zwischen dem Parietalbein und Occipitalbein beiderseits eine $2 \mathrm{~cm}$ breite, prall elastisch gefüllte Lücke; ebenso ist zwischen dem vorderen Rand des Parietalbeins und den hinteren Rand des Frontalbeins, sowie zwischen Parietal- und Schläfenbein eine fingerbreite Lücke geschaltet. Die große Fontanelle ist stark vorgebuchtet, prall elastisch, die Schädelknochen selber zeigen keine abnorme Weichheit. Tubera frontalia deutlich ausgebildet. Kopf wird in Opisthotonusstellung gehalten, die Wirbelsäule in der Lendengegend lordotisch gebeugt. Die Augen werden nicht geschlossen gehalten. Die Sklerae oberhalb der Corneae füllen den größten Teil der Lidspalte aus, während die Corneae nach unten gerichtet sind. Herztöne rein, Därme durch die Bauchdecken sichtbar. Am Kopf stark gestaute Venen.

27. IV. Ohne erkennbare Ursache Temperaturschwankung (zwischen 33 und $39^{\circ}$ ). Augenhintergrund normal (Professor Stock).

Es wird linkerseits Ventrikelpunktion vorgenommen. Anfangsdruck 210 bis $240 \mathrm{~mm}$; entleert $17 \mathrm{ccm}$ leicht sanguinolenter Flüssigkeit. Enddruck $180 \mathrm{~mm}$. Verband.

28. IV. Die Punktion wird gut überstanden. Es sickert noch immer Liquor nach. Der Kopfverband wird schnell naß. Der Kopf fühlt sich nicht mehr so prall gefült an wie früher. Der Kopfumfang zeigt keine meßbare Abnahme. 
Trotz 2 Wärmeflaschen abnorme niedrige Temperatur $\left(34,2-32^{\circ}\right)$. Appetit gering. Kein Erbrechen. Stuhl etwas dünn breiig, grüngelb. Kampfer. Gewicht $2600 \mathrm{~g}$.

29. IV. Sehr verfallenes Aussehen. Vita minima. Große Atmung. Mehrmals vom Personal schon tot geglaubt, immer wieder bessere Perioden. Temperaturen bis zu $29,4^{\circ}$. Wiederholt Kampfer.

30. IV. Exitus nach sehr langer Agone.

\section{Temperaturen:}

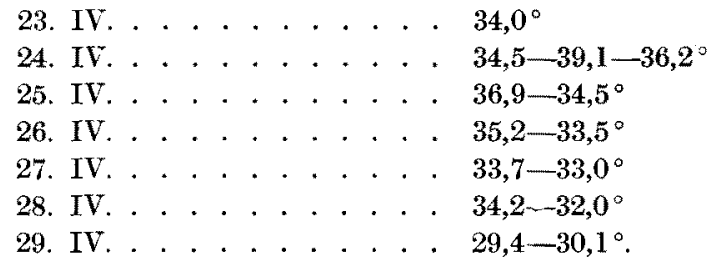

Die Sektion wurde 25 Stunden später von mir vorgenommen.

Obduktionsbefund: Leiche eines etwa 1 Monat alten Kindes männlichen Geschlechts. Totenflecke am Rücken, geringe grünliche Verfärbung der Bauchdecken in der rechten Unterbauchgegend. Körperbau schwächlich, sehr reduzierter Ernährungszustand. Hals ziemlich lang. Gesichtsschädel im Verhältnis zum übrigen Schädel relativ klein. Größter Schädelumfang $44 \mathrm{~cm}$. Größter Querdurchmesser (biparietal) $12 \mathrm{~cm}$; größter Längsdurchmesser ebenfalls $12 \mathrm{~cm}$. Alle Schädelknochen sind sehr leicht gegeneinander verschieblich. Die große Fontanelle bildet ein annähernd rhombisches Feld von $11 \mathrm{~cm}$ Länge und ebensolcher Breite. Zwischen beiden Stirnbeinen ist eine, nach dem Nasenbein zu sich verjüngende Furche von $3-4 \mathrm{~mm}$ Breite zu konstatieren. Stirnbeine beide rundlich gewölbt, stark vorspringend; die Bulbi scheinen infolgedessen sehr tief zu liegen, die oberen Lider schneiden etwa $1 \mathrm{~mm}$ oberhalb der Corneoskleralränder $a b$. Scheitelbeine nach hinten etwas über das Hinterhauptsbein hinübergeschoben. Auf der linken Seite des Kopfes, etwas links von der Mediane, fehlen die Haare im Bereich des Stirnbeins und eines Teils des Seheitelbeins; es mündet hier in die Haut eine ziemlich feine Kanüle, die nach innen in den Hirnventrikel führt. Keinerlei Entzündungserseheinungen in der Umgebung der Kanüle.

Die beiden Tibiae zeigen eine ganz unerhebliche, nach innen konkave Verbiegung.

Haut dünn, Muskulatur sehr spärlich, blaß.

Die Organe der Brust- und Bauchhöhle zeigen normale Verhältnisse, mit Ausnahme von den Lungen, in denen kleine atelektatische Herde aufgefunden werden.

Gehirn. Der Schädel wird nicht aufgesägt, sondern entsprechend den weitklaffenden Fugen zwischen den einzelnen Knochen aufgeschnitten. Im Sinus longitudinalis findet sich flüssiges Blut. Beim Wegklappen der Schädelknochen findet sich die Dura im Bereich der beiden Stirnbeine dünn, glänzend und glatt. Dagegen zeigt sie, nach ihrer Ablösung, im Bereich des linken Scheitelbeins eine eigentümliche marmorierte Färbung. Es finden sich hier unregelmäßige, inselartig angeordnete Flecken von leuchtend roter Farbe, an den Rändern geht diese 
Farbe zumeist in ein leuchtendes Gelb, Gelbbraun oder Sepiabraun über. Auf der rechten Seite sind diese Veränderungen an den Außenflächen der Dura nicht festzustellen. Beim Herausnehmen des Gehirns fließt aus dem Subduralraum eine geringe Menge klarer Flüssigkeit ab. Das Gehirn fühlt sich außerordentlich weich, schwappend an. Man fühlt im reehten Occipitallappen unter der hier papierdünnen Wand eine größere Cyste; von der Wand aus gehen hier feine, zerreißliche Stränge zur Dura. Sie werden vorsichtig durchtrennt, doch reißt hierbei die dünne Wand ein und es entleeren sich ca. $100 \mathrm{~cm}$ einer gelblichen, etwas trüben Flüssigkeit aus den Hirnhöhlen. Nach Wegnahme des Gehirns bemerkt man an der Innenfläche der Dura in den beiden mittleren Schädelgruben ähnliche Verhältnisse, wie im Bereich des linken Scheitelbeins. Die Dura zeigt nämlich auch hier eine rotbraune, stollenweise mehr gelbbraune Färbung. In diesen Partien finden sich der Dura aufgelagert, weiche, teils bröcklige, meist aber mehr schmierig breiige Massen von brauner oder braungelber Farbe. In stärkerem MaBe ist das der Fall im Bereich des linken Hinterhauptbeins; hier ist auf beiden Seiten der Dura die braunrote fleckige Färbung festzustellen, an der Innenfläche größere Mengen der schmierigen braunen Massen. Diese finden sich auch in der Gegend der rechten Hälfte des Hinterhauptbeins, wo vom Gehirn aus kleine strangförmige Verklebungen, ebenfalls von braungelber Farbe, mit der Dura bestehen.

Ein ähnlicher Befund ist noch am vorderen Rand der Sella turcica zu erheben, während nach hinten, am Clivus, derartige Veränderungen der Dura nicht bestehen. $\mathrm{Cm}$ das Chiasma optieum finden sich die bräunlichgelben Massen in größerer Menge.

Die Sinus der Hirnbasis enthalten flüssiges Blut. An den Knochen der Schädelbasis und des Schädeldachs sind nirgends Verletzungen festzustellen. Im Rückenmarkskanal findet sich ebenfalls vermehrte Flüssigkeit von leicht gelblicher Farbe. Zwischen Dura und Pia sind auch hier, da und dort in ausgedehntem Maße, bräunlichgelbe flüssige oder mehr breiige Massen zu konstatieren.

Die Pia zeigt ïberall, entsprechend den Stellen, wo an der Dura die Veränderungen festzustellen sind, eine bräunlichgelbe Färbung; dunkler ist diese über dem rechten Occipitallappen, wo Himrinde, Pia und Teile der Dura in eine mit Blut und gelbbraunen Brei durchtränkte Masse umgewandelt erscheinen. Da und dort ist die Pia hier auch eingerissen, man erkennt in dieser Gegend mehr blutig gefärbte Gerinnsel.

Das Gehirn selbst hat eine Länge von $15 \mathrm{~cm}$, eine Breite von $121 / 2 \mathrm{~cm}$; die größte Höhe beträgt $7 \mathrm{~cm}$. Die Hirnwindungen sind gut ausgebildet und ziemlich breit. Über der rechten Hemisphäre sind an der Konvexität weder Blutungen in der Pia noch irgendwelche Defekte der Hirnsubstanz zu erkennen.

Dagegen zeigen sich im rechten Occipitallappen und dem hinteren Teil des rechten Temporallappens ausgedehnte Veränderungen. Die Wand ist hier, entsprechend der größeren Blutung in der rechten hinteren Schädelgrube, aufs äußerste verdünnt, an einer Stelle eingerissen. Man erkennt, daß hier die Rinde mitsamt. der Pia völlig mit Blut und bräunlichen Massen durchtränkt ist. Die etwa hühnereigroße Cyste, die hier bei Herausnahme des Gehirns zu fühlen war, erwies sich als nichts anderes, als das sehr stark erweiterte rechte Hinterhorn. An der Spitze des rechten Temporallappens ist ebenfalls eine stärkere Braunfärbung und Durch-

Zeitschrift für Kinderheilkunde. O. II. 
tränkung der Pia vorhanden. Doch findet sich beim Einschneiden hier die Hirnrinde nicht verdünnt und keine Zerstörung in der Wand; erst nach hinten, gegen den Occipitallappen, wird sie immer dünner, schließlich fast papierdünn. Beim Einschneiden erkennt man, daß auch das Unterhorn des rechten Seitenventrikels sehr stark erweitert ist, so daß Unterhorn und Hinterhorn eigentlich nur eine große, weit kommunicierende Höhle bilden. Von dieser Höhle aus sieht man das Relief der Stirnwindungen deutlich durchschimmern.

Links findet man entsprechend dem Lobus parietalis superior und inferior in der Pia ausgedehnte Blutung und Zerreißung; reichlich bräunliche schmierige Massen zwischen Pia und der hier äußerst dünnen Hirnrinde. Man erkennt in diesem Bereich eine recht unregelmäBig begrenzte bucklige, jedoch nicht sehr in die Tiefe reichende, also mehr spaltenförmige, etwa 5-Markstückgroße Höhle, deren obere Decke von der zerstörten und stellenweise eingerissenen Rinde und Pia gebildet ist; in der Höhle kleine Fetzen zertrümmerter Hirnsubstanz. Der Boden dieser Höhle ist an der dünnsten Stelle $21 / 2 \mathrm{~mm}$ dick. Hier bildet der Boden das Dach des erheblich erweiterten linken Seitenventrikels. Dieser kommuniziert durch das Foramen Monroi, das einen Durchmesser von ca. $8 \mathrm{~mm}$ hat, mit dem rechten Seitenventrikel. Die Innenwand der Hirnventrikel erscheint fast ganz glatt, nur da und dort ganz fein warzig. Der Balken ist papierdünn, völlig durchsichtig. In der Wand der Ventrikel gewahrt man von innen gesehen, wenig gefüllte Venen. Im Ventrikelinhalt keine Trümmer von Himsubstanz. Unterund Seitenhorn beiderseits gleich stark erweitert.

An den Gefäßen und Nerven der Hirnbasis sind keine Veränderungen festzustellen, beide Optici drehrund, ohne Differenz zwischen rechts und links.

Im Bereich der linken Fossa Sylvii zeigt die Pia etwas stärkere bräunliche Färbung.

Am Kleinhirn sind makroskopisch keine Veränderungen zu erkennen. Am Rückenmark (abgesehen von den Blutungen in den Häuten) ebenfalls keine Veränderungen.

Die mikroskopische Untersuchung von Leber, Nieren, Milz ergab durchaus normale Befunde. In der Lunge finden sich ausgedehnte atelektatische Partien. Hier im Lumen der Alveolen neben zahlreichen abgestoßenen Alveolarepithelien auch rote Blutkörperchen, Leukocyten sowie grünlich-braungefärbte Massen, die zum Teil in Zellen aufgenommen sind (aspirierte Mekoniummassen). Leider wurde keine eingehendere histologische Untersuchung der Lungen vorgenommen.

Die bräunlichen schmierigen Massen auf der Dura und Pia bestehen mikroskopisch aus roten Blutkörperchen und großen Mengen von Blutpigment (Hämosiderin); ganz vereinzelt finden sich auch Hämatoidinkristalle.

Die Hirnhäute sind im ganzen etwas verdickt. Im Subduralraum, in den Maschen der Arachnoidea und auf der Pia finden sich große Mengen freiliegenden, körnigen Pigmentes, das die Eisenreaktion gibt; an wenigen Stellen, wo ganz dichte Pigmentklumpen vorhanden sind, sind auch rhombische Hämatoidin. kristalle aufzufinden. Aber auch in den Bindegewebszellen der Hirnhäute finden sich noch erhebliche Mengen von körnigem, braunem Blutpigment. In der Hirnrinde sind neben kleinen Blutungen ebenfalls noch Massen von Hämosiderin, teils frei, teils in Zellen aufgenommen, anzutreffen.

Analoge Befunde sind am Rückenmark zu konstatieren. Hier ist noch etwas 
mehr freies Blut zwischen den Häuten, vor allem der Dura vorhanden; besonders reichliche Massen von körnigem Blutpigment findet man in den Scheiden der Rückenmarksnerven. Die Substanz des Rückenmarks selbst ist frei von Veränderungen. Insbesondere fehlen hier, wie auch am Gehirn, jegliche Erscheinungen entzündlicher Prozesse.

Die Plexus chorioidei der Seitenventrikel sind etwas verdickt. In den Bindegewebszellen der Plexus sind auffallend große Klumpen von Hämosiderin nachzuweisen; Hämatoidin findet sich nirgends. Sowohl die Epithelzellen als die Bindegewebszellen der Plexus sind größtenteils fetthaltig, im Bindegewebe findet sich das Fett in feinen Tröpfchen. In manchen Zellen sowohl Fett als Blutpigment nachzuweisen.

Bei der Deutung dieses Falles stößt man leider auf nicht unbeträchtliche Schwierigkeiten. Baß die Blutungen im Gehirn und seinen Häuten auf ein Trauma zurückzuführen sind, wird allerdings nicht zu bestreiten sein, da Anhaltspunkte für eine andere Ätiologie zunächst fehlen; vor allem fehlen jegliche entzündliche Prozesse, wie die histologische Untersuchung ergab; ebenso lag weder Porencephalie, noch Mikrogyrie oder ähnliches vor. Es fragt sich demnach: wann hat ein Trauma stattgefunden? und ferner: was hat der Hydrocephalus mit den anderen Veränderungen zu tun?

Die Anamnese kann uns hierüber nicht ausreichenden AufschluB geben. Es steht soviel fest: Das Kind ist von gesunder Mutter nach 20 stündiger Geburtsdauer geboren: für eine Erstgebärende, dazu bei etwas platten Becken, ist diese Zeit durchaus nicht ungewöhnlich lang. Die Geburt erfolgte spontan, und es hat dabei jedenfalls kein nachweisbares Trauma stattgefunden. Im Protokoll ist eigens erwähnt, daß eine größere Geburtsgeschwulst sich nicht vorfand. Nun sind die Angaben über die Schädelmasse des Kindes insofern auffällig, als der frontooccipitale Durchmesser und. Umfang $2 \mathrm{bzw} .4 \mathrm{~cm}$ mehr als normal beträgt, während der biparietale normal ist. Da es aber nicht ausgeschlossen erscheint, daß bei der Messung ein Irrtum untergelaufen sein kann, möchte ich auf diese Maße nicht zu viel Gewicht legen. Der fronto-occipitale Durchmesser ist bei Entlassung des Kindes zu 12,5 cm angegeben, also um $11 / 2 \mathrm{~cm}$ weniger als bei der Geburt: wenn nun tatsächlich, wie bemerkt, eine stärkere Geburtsgeschwulst nicht bestanden hat, so wäre das schwer zu erklären. Jedenfalls ist aber - und das scheint besonders bemerkenswert - bis zur Entlassung von Mutter und Kind etwa 8 Tage lang kein abnormes Verhalten des Kindes bemerkt worden, und keine Symptome einer cerebralen Affektion, wie das bei dem später mitzuteilenden Falle so deutlich war. 
Erst etwa 3 Wochen nach der Geburt soll, nach den Angaben der Mutter, der Kopfumfang des Kindes zugenommen haben. Über ein Trauma in dieser Zeit ist nichts bekannt; es wäre aber ganz wohl denkbar, daß ein solches stattgefunden hat, aber, vollends wenn etwa fahrlässiges Verhalten der Mutter vorgelegen hat, verschwiegen wurde. Es erscheint nach den ausgedehnten Blutungen im Gehirn und seinen Häuten ganz undenkbar, daß diese keine klinischen Symptome gemacht haben sollten. Solche wären aber, falls etwa ein Geburtstrauma vorlag, bei der Beobachtung in der geburtshilflichen Klinik nicht unbemerkt geblieben. Demnach wird wohl der Zeitpunkt des Traumas zusammenfallen mit der Zeit, von welcher ab, wie die Anamnese angibt, ein allmähliches Zunehmen des Kopfumfanges, und damit auch schwere Krankheitssymptome manifest wurden: also erst nach der Entlassung aus der Klinik. Der Zeitpunkt der Entstehung der Blutungen wird demnach etwa in die 2 . bis 3 . Woche nach der Geburt, also 2 bis 3 Wochen vor dem Tode des Kindes zu legen sein. Der histologisehe Befund läßt sich mit dieser Annahme wohl in Einklang bringen. Es fand sich zwischen den Hirnhäuten noch flüssiges Blut neben großen Mengen von körnigem Blutpigment. Die Hämatoidinkristalle waren nur spärlich. Bei experimentell erzeugten Blutungen sah Dürck ${ }^{1}$ ) Hämatoidinkristalle erstmals nach 18 Tagen auftreten: nimmt man diesen Zeitraum als Minimum an, so würde das mit unserer Annahme ganz gut übereinstimmen.

An sich läge es ja natürlich viel näher, die beobachteten Veränderungen auf ein Geburtstrauma zu beziehen, wie das ja auch in so vielen Fällen zutrifft, mindestens angenommen wird. Aber ich glaube, nach den anamnestischen und klinischen Daten ist diese Annahme nicht erlaubt. Man müßte dann annehmen, die Blutungen seien ganz allmählich erfolgt und hätten also während eines längeren Zeitraums stattgefunden und so erst allmählich zu klinischen Erscheinungen und Hydrocephalus geführt. Das wäre denkbar, aber unwahrscheinlich. $\mathrm{Zu}$ berücksichtigen ist noch, daß zu den schon vorhandenen Blutungen in letzter Zeit natürlich noch neue gekommen sein können. Dazu kann z. B. die Punktion Anlaß gegeben haben. Durch solche hinzugekommene Blutungen kann das Bild etwas verwischt werden, was bei der Bewertung der Lokalisation der Blutungen noch mit zu berücksichtigen ist.

1) Dürck , Beitrag zur Lehre von den Veränderungen und der Altersbestim. mung von Blutungen im Zentralnervensystem. Virchows Archiv 130. 
Endlich kann noch in Frage kommen, ob nicht schon kongenital ein Hydrocephalus bestanden hat. Dafür liegt kein Anhaltspunkt vor, und die bei der Geburt und 8 Tage nachdem aufgenommenen, wenn auch möglicherweise nicht absolut exakten Maße sprächen auch dagegen. Es könnte sich dann auch nur um einen wenig ausgesprochenen Hydrocephalus bandeln, der dann eine leichtere Zerreißbarkeit der Gefäße usw. möglich erscheinen ließe: aber Anhaltspunkte oder Beweise für diese Annahme liegen m. E. nicht vor.

Vielwahrscheinlicher wird eine umgekehrte causale Beziehung aufzustellen sein, nämlich eine sekundäre Entstehung des Hydrocephalus durch die primäre Blutung. Darauf soll jedoch erst weiter unten eingegangen werden: zunächst soll noch über einen 2 . Fall berichtet werden, der mit dem oben mitgeteilten so vieles gemein hat, daß die Besprechung verschiedener Punkte für beide Fälle gemeinsam erfolgen kann.

\section{Fall. Julius D., 31/2 Monate alt.}

Geburtsgeschichte: Die Mutter L. D. ist 29 Jahre alt; 2. para. Die erste Geburt 1904 wurde wegen engen Beekens durch Wendung und Extraktion beendigt. Das Kind starb bei der Geburt.

Am 17. Mai 1909 begannen die Wehen, 2 Uhr nachts. Morgens kommt die Frau mit starken Wehen in die Frauenklinik. Bei der Untersuchung wird festgestellt, daß eine erste Schädellage vorliegt, Kind lebt. Der Kopf sitzt über dem Becken. Das Becken ist platt, rhachitisch; die Conjugata vera wird zu $7,3 \mathrm{~cm}$ bestimmt (also platt-rhachitisches Becken 3. Grades). Um 9 Uhr morgens Blasensprung, Abgang von Mekonium. Muttermund handtellergroß. Die Nabelschnur ist vorgefallen, pulsiert; Herztöne zwischen 120 und 180 . Es wird daher 10 $1 / 4$ Uhr Sectio caesarea abdominalis vorgenommen, unter Lumbalanästhesie mit Stovain nach vorhergehender Injektion von 0,0003 Scopolamin und 0,01 Morphium. Ideale Anästhesie. Die Operation verläuft ungestört und wird in ty* pischer Weise vorgenommen.

Das mittels Kaiserschnitt zur Welt gebrachte Kind, männlichen Geschlechts, ist zunächst asphyktisch. Auf thermische Hautreize und Herzmassage erfolgt nach 5 Minuten die erste Atmung.

Gewicht des Kindes $2950 \mathrm{~g}$.

Die Maße des Kopfes sind die folgenden:

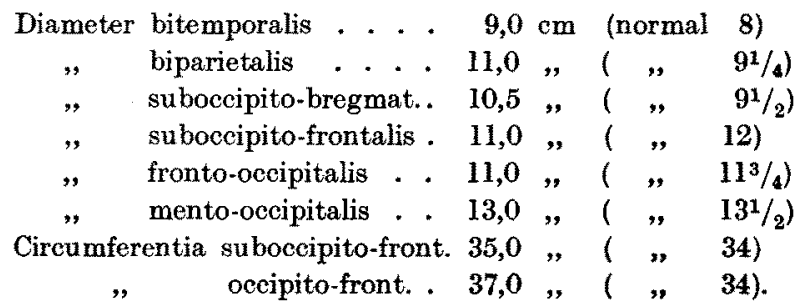


Am 18. V. machte das Kind sehr schlechten Eindruck; cerebrale Erscheinungen, Handzuckungen, starke Spannung der großen Fontanelle.

Am 19. V. wird das Kind aufgegeben; absoluter Verfall, starke Cyanose, diffus feinblasiges Rasseln über beiden Lungen. In den nächsten Tagen erholt sich das Kind langsam, dabei bemerkt man ein deutliches Anschwellen des Kopfes. Die Nähte werden weiter, die Fontanellen gespannt und groß. Bei der Entlassung des Kindes (1. VI.) besteht deutlicher Hydrocephalus.

Das Kind wird nicht gestillt.

Bei der Entlassung Kopfumfang $41 \mathrm{~cm}$ (bei der Geburt $35 \mathrm{~cm}$ ). Sagittaler Durchmesser 13,5 (11,0 cm), biparietaler Durchmesser $12,0(11,0 \mathrm{~cm})$.

Gewicht $2800 \mathrm{~g}$.

Am 1. IX. wird das Kind in das Hildaspital aufgenommen. Nach den anamnestischen Angaben soll es eine Zeitlang Durchfall gehabt haben, hat nicht getrunken. Fieber und Erbrechen soll nicht bestanden haben. Gewicht bei der Aufnahme $3140 \mathrm{~g}$.

Befund: Unruhiges, blasses Kind, in sehr dürftigem Ernährungszustand. Iringe $51 \mathrm{~cm}$, Kopfumfang $41 \mathrm{~cm}$, Brustumfang $42 \mathrm{~cm}$. Haut welk, Bauchdecken stark gespannt. Kopf in Opisthotonushaltung, kann nicht auf die Brust gebeugt werden. Halsvenen stark gestaut. Große Fontanelle $2 \mathrm{~cm}$ lang und breit, gespannt. Bulbi schauen nach unten, meist ist nur Sklera zu sehen.

Herztöne sehr langsam, Töne rein, regelmäßig. Hypertonie der Extremitäten; sie sind kühl, cyanotisch.

2. IX. Lumbalpunktion: $9 \mathrm{ccm}$ wasserklaren Liquors entleert. Anfangsdruck $150 \mathrm{~mm}$, Enddruck $100 \mathrm{~mm}$.

Am 3. IX. morgens Exitus.

Temperaturen: 1. IX. $35^{\circ}$; 2. IX. morgens $33^{\circ}$, später $34,5^{\circ}$.

Die Sektion des Kindes konnte ich am 3. IX. 3 Stunden nach dem Tode des Kindes ausführen.

Sektionsbefund: Leiche eines etwa 4 Monate alten, mäßig kräftig gebauten Kindes männlichen Geschlechts. Keine Starre, etwas Totenflecke am Rücken. Ziemlich reduzierter Ernährungszustand. Haut welk. Keine sichtbaren Verletzungen oder Anomalien der Haut. Pupillen ziemilich weit. Stirnschädel sehr stark gegen den Gesichtsschädel vortretend, Stirn hoeh, etwas eckig. Kopfumfang $42 \mathrm{~cm}$. Ausgeprägte Stirn- und Scheitelhöcker. Große Fontanelle von etwa entsprechender Größe. Schädelknochen recht dick und fest.

Nach Wegnahme des Schädeldaches läßt sich an der AuBenfläche der Dura nichts Bemerkenswertes erkennen. Im Sinus longitudinalis flüssiges Blut. Aus dem Subduralraum entleert sich eine reichliche Menge klarer, leicht bräunlich gefärbter Flüssigkeit. Die Piavenen wenig gefüllt, die Stirnwindungen durch die Dura durehschimmernd.

Es zeigt sich, daB an der Basis des Gehirns, insbesondere im Gebiet der linken hinteren und mittleren Schädelgrube, im geringen Grad auch in der rechten hinteren und mittleren und in ganz geringem Grad auch in den vorderen Schädelgruben, die Dura verdickt ist. Sie hat hier eine braungelbe, zum Teil fast rostige Farbe, beim Durchschnitt findet man überall breiige, eingedicktem Blut ähnliche Massen, die die Dura durchsetzen und auch auf ihrer Innenfläche aufgelagert sind. An diesen Stellen findet sich nun die Pia mater vielfach mit der Dura mebr 
oder weniger fest verklebt, teils durch kleine bindegewebige Stränge, teils nur durch breiige, auch mehr schmierige oder fast flüssige bräunliche Massen. Die Pia mater selbst zeigt hier überall eine bräunliche oder braungelbe, rostige Farbe. Vom Chiasma rückwärts, vorzugsweise auf dem Pons und nach den Brachia pontis za, ist die Farbe der Pia annähernd ockergelb, ebenso in der Gegend der Olive und über beiden Kleinhirnhemisphären. Auch in der Fossa Sylvii beiderseits etwas Verfärbung der Pia und geringe Mengen bräunlicher Massen zwischen den weichen Hirnhäuten. Dasselbe ist der Fall an der Spitze der Temporallappen, links nur an der Spitze und gegen die Pedunculi cerebri zu; rechts dagegen wird die Braunfärbung nach hinten gegen den Oceipitallappen zu immer intensiver. Starke Gelbbraunfärbung der Pia und Durchtränkung der weichen Hirnbäute mit gelbbraunen Massen am Kleinhirn, zwischen den Floceulis und dem Austritt der 8.-10. Hirnnerven. An der Oberfläche des Gebirns ist die Pia durchaus glatt, dünn, die Venen mäßig gefüllt; ebenso an der Basis über den beiden Frontallappen.

Im Gebiet des rechten Schläfenlappens und Occipitallappens ist die Rinde des Gehirns äußerst dünn. Bẹ Herausnahme des Gehirns reißt hier die Pia und Hirnrinde ohne weiteres ein, es entleert sich aus dem Innern, anscheinend aus den Hinter- und Unterhörnern, sehr reichlich klare Flüssigkeit von gelblichbrauner Farbe.

An den Arterien der Hirnbasis sind, keine pathologischen Veränderungen festzustellen; die Communicans posterior ist links wesentlich stärker als rechts. In den Sinus der Hirnbasis flüssiges Blut, an den Knochen der Schädelbasis sind keinerlei Veränderungen festzustellen. Die Nervi optici beiderseits gleich dick; etwas abgeplattet.

Das herausgenommene Gehirn zeigt folgende Maße:

$$
\begin{aligned}
& \text { Länge. . . . . . . . 15,5 cm } \\
& \text { Breite. . . . . . . . 13,5, } \\
& \text { Höhe . . . . . . . . . . 6,5, }
\end{aligned}
$$

Etwa entsprechend dem Verlauf der Arteria cerebri posterior auf der rechten Seite, im vorderen Teil mehr nach der Spitze des Temporallappens zu, ist auf eine Strecke von mehreren Zentimetern die völlig mit Blut und schmierigen braungelben Massen durchtränkte Pia und Hirnrinde bei Herausnahme des Gehirns eingerissen. Man gelangt in eine annähernd eiförmig gestaltete Höhle, deren innere Wandung fast ganz glatt erscheint. Nur findet sich hier und da der Wand innen aufgelagert, gelbliches oder bräunliches Pigment in geringen Massen. Die beschriebene Höhle reicht nach hinten bis zur Spitze des Occipitallappens: hier ist die äußere Wandung der Höhle von der Hirnrinde gebildet und etwa $4 \mathrm{~mm}$ dick. Es erweist sich, daB die Höhle nichts anderes ist als das stark erweiterte rechte Hinter- und Unterhorn, wie an dem Plexus chorioideus, der von vorn her in die Höhle hereinragt, leicht ersichtlich ist.

Der Balken stellt eine im Maximum $11 / 2 \mathrm{~mm}$ dicke, nach oben etwas konvex vorgebuchtete Platte dar. Nach seiner Durchtrennung sieht man, daß beide Seitenventrikel, Vorder-, Unter- und Hinterhorn beiderseits gleichmäBig erweitert sind. Die Konturen der Stammganglien treten deutlich hervor, diese wölben sich konvex in die Höhle hinein vor. Die Plexus chorioidei beiderseits ziemlich klein und dünn, hier und dort erkennt man an ihnen festhaftend gelbbraune 
Schüppchen. Ebenso in der Wand der Seitenventrikel an manchen Stellen gelbbraune Verfärbungen der Wand.

Während die linke Hälfte des Kleinhirns normale Konfiguration zeigt, finden sich in der rechten Hälfte ausgedehnte Veränderungen. Es besteht hier ein Defekt, der fast den ganzen Lobulus semilunaris inferior et biventa betrifft, während die Tonsille unverändert erscheint. Entsprechend dem Defekt findet sich Pia and Kleinhirnrinde in eine weiche, fast breige braune Masse umgewandelt. Beim Durchschnitt dureh den Defekt sieht man, daB im Zentrum hier ein annähernd rautenförmiger Hohlraum gebildet ist, der nach außen in größter Ausdehnung von der Kleinhirnrinde begrenzt wird. Die Marksubstanz erscheint fast ganz zerstört, oder, wo noch erhalten, mit bräunlichen Massen durchtränkt. An einer Stelle ist eine nur papierdünne Begrenzung des Hohlraumes vorhanden, er ist hier nach außen anscheinend nur durch die mit Blutpigment durchtränkte rostfarbige Pia abgeschlossen.

An der Medulla und am Rückenmark auf dem Durchschnitt nichts Pathologisches zu erkennen; aus dem Subduralraum entleert sich auch hier leicht bräunlich gefärbter Liquor.

Die übrige Sektion war nicht gestattet. Es konnten nur durch einen kleinen Schnitt am Halse die Halsorgane entfernt werden. Auf der Zunge, der linken Tonsille, in den unteren Teilen des Pharynx und im Anfangsteil des Oesophagus finden sich kleine, etwas schmutzigweiße bis grauweiße, nicht sehr festhaftende Beläge (Soor). Beide Schilddrüsenlappen etwas vergröBert, zeigen Bildung deutlicher kleiner Knoten. Im Larynx nichts Besonderes.

Herz und Lungen (soweit sie von dem Schnitt aus herausgeholt werden konnten) weisen durchaus normalen Befund auf.

Die Sektionsdiagnose lautete demnach: Alte ausgedehnte Blutungen der Pia und Dura mater, besonders im Bereich der linken mittleren Schädelgrube. Blutungen im rechten Schläfenlappen und in der rechten Kleinhirnhemisphäre. Hydrocephalus internus und externus. Atrophie. Struma congenita. Soor der Zunge, des Rachens, des oberen Teiles des Oesophagus.

Die mikroskopische Untersuchung ergab: Die Dura ist etwas verdickt. An ihrer äußeren und an ihrer inneren Oberfläche findet man große Massen von goldgelbem oder etwas mehr braunem körnigem Pigment, das oft in großen Klumpen zusammengeballt ist und Eisenreaktion gibt. Inmitten der großen Haufen des Pigments finden sich auch Kristalle von Hämatoidin. Auch in den Gewebszellen der Dura, besonders in den Randpartien, in der Mitte am wenigsten, trifft man Pigment, teils frei im Gewebe in Spalten, teils in Zellen aufgenommen; da und dort sieht man inmitten größerer Herde von Hämosiderin auch kleine Hämatoidinkristalle. Die Bindegewebszellen der mittleren Duraabschnitte sind größtenteils fetthaltig, die mit Pigment beladenen Zellen fettfrei. Das Gewebe der Dura ist im ganzen sehr kernarm. Unter der Dura, in den Maschen der Arachnoidea und unter der Pia findet man überall Blut und Blutpigment. Kristalle fehlen hier. Auch in den äußeren Schichten der Hirnsubstanz finden sich mit Pigment beladene Zellen; an manchen Stellen ist die ganze Hirnrinde diffus pigmentiert. Die Pigmentkörner sind hier jedoch kleiner als in und zwischen den Hirnhäuten.

Das Ventrikelependym ist nur zum Teil erhalten. Dicht unter dem Ependym 
erkennt man an mehreren Stellen wieder eine diffuse Pigmentierung der Zellen; das körnige Pigment findet sich hier nur in Zellen, nicht frei im Gewebe. Da, wo das Ependym zerstört ist, ist vielerorts ein sehr gefäßreiches junges Bindegewebe anzutreffen, dessen Zellen zum Teil ebenfalls Blutpigment aufgenommen haben.

Die Plexus der Seitenventrikel sind zartwandig, in den Buchten zwischen dem Epithel findet man viel Pigment, im Plexusgewebe selbst nur an wenigen Stellen.

Auch in und zwischen den Häuten des Rückenmarks ist körniges Pigment, vorhanden, Kristalle fehlen. Entzündliche oder anderweitige pathologische Prozesse fehlen durchaus.

Kleinhirn, rechte Hälfte: Hirnhäute ehenfalls mit Blutungen und Blutpigment durchsetzt. Die Marksubstanz des Kleinhirns ist fast völlig von Blutpigment durchsetzt; es liegt hier in großen Schichten so dick, daß vielfach von der Marksubstanz überhaupt nichts mehr zu sehen ist. Fast alles Pigment liegt frei im Gewebe, nur da und dort sieht man Capillaren dazwischen hindurchziehen. Sehr sehön ist die Ablagerung von Pigment überall in der Umgebung der Gefäße zu sehen. Von der Rindenschicht sind große Teile zerstört und vor allem die granulierte Schicht, die auf kleinen Strecken ganz fehlt. Die äußere Körnersehicht ist meist noch gut zu erkennen, die gangliöse Schicht erscheint zum Teil etwas verschmälert. Blutpigment ist auch in den Rindenschichten in großer Menge vorhanden, besonders in der äußeren Körnerschicht, doch nicht so reichlich wie im Mark. Wo größere Partien der Körnerschicht zerstört sind, sieht man außer freiem Pigment auch große spindlige, mit Pigment beladene Gliazellen.

Zusammengefaßt wäre über diesen Fall zu sagen: Bei einer 2. para mit erheblich verengten Becken (Conjugata vera von $7,3 \mathrm{~cm}$ ) wird in der Eröffnungsperiode wegen Nabelschnurvorfalls und Abgang von Mekonium Kaiserschnitt vorgenommen und ein ausgetragenes Kind zur Welt gebracht. Das Kind bietet am Tag nach der Geburt schwere cerebrale Erscheinungen; es wird allmähliches Zunehmen des (bei der Geburt schon etwas großen) Kopfumfanges und bei der Entlassung am 15. Tage deutlicher Hydrocephalus konstatiert. Im Alter von $3^{1 / 2}$ Monaten stirbt das Kind unter ausgesprochenen cerebralen Erscheinungen. Die Obduktion ergibt Hydrocephalus, ausgedehnte alte Blutungen in den Hirnhäuten, und im rechten Schläfen- und Hinterhauptlappen sowie im Kleinhirn.

Der Zeitpunkt der Entstehung dieser Blutungen läßt sich in diesem Falle ziemlich genau feststellen. Die Angaben der Klinik zeigen uns, daß die Krankheitserscheinungen (cerebrale Erscheinungen, Zuckungen) wie auch die objektiven Erscheinungen der Volumszunahme des Kopfes gleich nach der Geburt eingesetzt haben. Es ist deshalb in diesem Fall an ein Geburtstrauma als Ursache dieser Blutung zu denken, wenn nicht etwa dieses Trauma in noch frühere Zeit zurückzuverlegen sein sollte. Zum mindesten hat hier am Tage nach der Ge- 
burt schon ein intrakranielles Hämatom vorgelegen; darauf deutet die Spannung der großen Fontanelle hin. Nach der Lage der Dinge ist nun aber nicht so leicht zu ersehen, wann bei der Geburt ein stärkeres Trauma auf den kindlichen Schädel eingewirkt hat. Die Möglichkeit dazu war allerdings bei dem stark verengten Becken gegeben; immerhin war der Kopf ja noch nicht ins Becken eingetreten, als aus dringender Indikation der Kaiserschnitt vorgenommen wurde. Trotzdem werden wir annehmen müssen, daß hier eine Verletzung infolge der Geburt stattgefunden hat, als die Wehen den Kopf in das hochgradig verengte Becken einzupressen versuchten.

Wie verhält es sich nun aber mit dem Hydrocephalus? Hier gelten dieselben Utberlegungen wie beim Fall Nr. 1. Wir dürfen annehmen, der Hydrocephalus bestand ante partum noch nicht. Dagegen hat er sich im Anschluß an die Geburt entwickelt, wie aus den klinischen Beobachtungen hervorgeht. Nehmen wir nun, wie oben ausgeführt, an, das in Frage stehende Trauma sei ein Geburtstrauma gewesen, so liegt es nun nahe, auch den Hydrocephalus in ursächliche Beziehung zu der (traumatischen) Blutung zu bringen.

Denn alle die anderen Ursachen, die man sowohl für die Entstehung eines Hydrocephalus (congenitus oder acquisitus) verantwortlich $\mathrm{zu}$ machen pflegt, fehlen hier. Leider wissen wir ja über diese ätiologischen Faktoren recht wenig. Aber wir können jedenfalls entzündliche Prozesse, etwa an dem Ventrikelependym oder den Plexus choriodei hier ausschließen: denn es fanden sich keine Spuren von solchen. Da nun anderseits in unseren beiden Fällen gleichzeitig zweierlei pathologische Veränderungen vorhanden sind: Blutungen und Hydrocephalus, liegt es ja schon von vornherein nahe, diese beiden in ursächlichen Zusammenhang zu bringen. Nach dem Gesagten wird das nur so möglich sein, wenn wir in den Blutungen das Primäre, im Hydrocephalus das Sekundäre erblicken. Der Hydrocephalus wäre demnach in unseren Fällen ein erworbener, ein traumatischer. Der Mechanismus wäre so zu deuten, daß durch die ausgedehnten Blutungen Verhältnisse geschaffen waren, die zu einer Erschwerung des Liquorabflusses bzw. des venösen Abflusses führten. Für die Annahme einer vermehrten Liquorbildung liegt kein Grund vor. Es kam ferner als unterstützendes Moment hinzu, daß durch ausgiebige Zerstörung von Teilen der Ventrikelwände die Widerstandsfähigkeit dieser Teile gelitten hatte: ein Faktor, der nach Weber ${ }^{1}$,

1) L. W. Weber, Utber erworbenen Hydrocephalus internus. Archiv f. Psych. 41. 
ebenfalls für die Entstehung des erworbenen Hydrocephalus zu berücksichtigen ist. Da nun die Resorption von Blutergüssen doch nur recht langsam vor sich geht, ist es ganz verständlich, daß ein Ausgleich zwischen diesem Druck von außen und von innen nicht erzielt wurde, daß vielmehr bei der dauernden Produktion des Liquors und der dauernd behinderten Abflußmöglichkeit der hydrocephalische Prozeß fortschreiten mußte. Es sei hier auch noch hingewiesen auf die im Falle 2 eigens erwähnte venöse Stauung (in den Halsvenen).

Die mikroskopische Untersuchung unserer Fälle hat gezeigt, daß die Blutungen erstens schon längere Zeit vor dem Tode bestanden haben müssen, daß schon ein gewisser Teil des ergossenen Blutes, vor allem bei Fall Nr. 2, resorbiert worden ist. Doch soll auf diesen Prozeß nicht weiter eingegangen werden. Nur soll noch auf einen Punkt beim Fall Nr. 2 hingewiesen sein: dạs ist die ausgedehnte Zerstörung von Kleinhirnsubstanz, die makroskopisch und mikroskopisch zu erkennen ist.

Eine so ausgedehnte Läsion ist an diesem Ort immerhin recht selten. Das sich darbietende Bild erinnert fast schon an einen porencephalischen Defekt; jedenfalls hätte sich bei einer Heilung ein solcher hier gebildet, ebenso an den anderen affizierten Abschnitten des Gehirns. Fs finden sich in der Literatur eine ganze Reihe von Fällen, bei denen ein porencephalischer Defekt auf ein Trauma, insonderheit auf ein Geburtstrauma zurückzuführen war, so z. B. in den Fällen von v. Kahlden ${ }^{1}$ ), Richter ${ }^{2}$ ), v. Monakow ${ }^{3}$ ). Traumatische Hirnblutungen bei der Geburt sind ja auch nicht selten. Darauf hat besonders Gowers ${ }^{4}$ ) aufmerksam gemacht. Auch bei Bednar ${ }^{5}$ ) ist darüber im Jahre 1850 schon sehr zutreffend berichtet. Aber daB solche Blutungen zu einem so hochgradigen Hydrocephalus, und durch diesen schließlich zum Tode führen, ist offenbar ein ungewöhnliches Ereignis. Geringfügige Traumen werden eben meist nur zu unbedeutenden Blutungen führen, es kann wohl auch ein geringer Grad von Hydrocephalus eintreten: aber solche Fälle heilen eben meist aus, oder führen etwa zu einem porencephalischen Defekt. Daß ein Hydrocephalus infolge traumatischer Geburtsblutungen (bei Tentoriumzerreißung) eintreten kan n,

1) C. v. Kahlden, Über Porencephalie. Zieglers Beiträge 28.

2) A. Rich ter, Über die Porencephalie. Centralbl. f. Nervenheilk, u. Psych. 21.

3) C. v. Monakow, Archiv f. Psych. 27, 386ff.

4) W. R. Gowers, Handbuch der Nervenkrankheiten 2, $406 \mathrm{ff}$.

5) A. Bednar, Die Krankheiten der Neugeborenen und Säuglinge. II. Wien 1850 . 
262 W. Fischer: Hydrocephalus nach Dura- und Gehirnblutungen bei Sauglingen.

hat Beneke übrigens in seiner jüngsten Veröffentlichung hervorgehoben. Auch Ha mburger (Zeitschrift für Heilkunde Bd. 26, S. 188), führt in einem Falle die Entstehung des Hydrocephalus auf ein Geburtstrauma zurück.

Wo nun in unseren Fällen das Trauma eingewirkt hat, läßt sich nicht feststellen. Es ist bemerkenswert, daß in unseren beiden Fällen an mehreren Stellen des Gehirns, nicht etwa bloß auf einer Seite übrigens nie an symmetrischen Stellen - eine blutige Zertrümmerung stattgehabt hat. (Nach der Kindslage vor der Geburt, 1. Schädellage könnte man vorwiegend rechts über dem Scheitellappen ein Hämatom erwarten: was nicht ganz zutrifft, doch war in beiden Fällen der rechte Temporal- und Occipitallappen betroffen).

Endlich soll noch auf einen Punkt hingewiesen werden. In beiden Fällen wurden ganz abnorm niedrige Temperaturen bei der klinischen Beobachtung festgestellt; im Falle 1 herab bis zu $29,4^{\circ}$, im 2 . bis zu $33^{\circ}$. Hierin ist offenbar der Ausdruck des völligen Versagens der Regulation der Körpertemperatur zu erblicken. Das zeigt sich auch an den Temperaturschwankungen im Laufe eines Tages, z. B. bei Fall 1 am 24. 4. die Werte: morgens $34,5^{\circ}$ mittags $39,0^{\circ}$, abends $36,2^{\circ}$. Diese Unfähigkeit der Temperaturregulierung ist offenbar auf die cerebralen Läsionen zurückzuführen; wie ja z. $B$. bei Hirnblutungen auch die Temperatur meist eine Stunde nach dem Insult auf $35,5^{\circ}$ und weniger herabsinken kann, um nachher wieder normale oder erhöhte Werte anzunehmen. 